\title{
UNIQUENESS OF TRACES ON LOG-POLYHOMOGENEOUS PSEUDODIFFERENTIAL OPERATORS
}

\author{
C. DUCOURTIOUX ${ }^{\bowtie}$ and M. F. OUEDRAOGO
}

(Received 27 July 2010; accepted 16 December 2010)

Communicated by S. Rosenberg

Dedicated to Alan Carey, on the occasion of his 60th birthday

\begin{abstract}
We show how to derive the uniqueness of graded or ordinary traces on some algebras of logpolyhomogeneous pseudodifferential operators from the uniqueness of their restriction to classical pseudodifferential ones.
\end{abstract}

2010 Mathematics subject classification: primary 47G30; secondary 58J40.

Keywords and phrases: log-polyhomogeneous pseudodifferential operators, traces.

\section{Introduction}

We consider a closed connected Riemannian manifold $M$ of finite dimension $n$ and a finite rank hermitian vector bundle $E$ over $M$. A pseudodifferential operator (PDO) acting on smooth sections of $E$ is called classical (or polyhomogeneous) (see [13]) if, locally, its symbol is classical, that is, it admits an asymptotic expansion in positively homogeneous components.

A pseudodifferential operator $L$ acting on smooth sections of $E$ is called logpolyhomogeneous if, locally, its symbol has the form

$$
a_{k}(x, \xi) \log ^{k}|\xi|+a_{k-1}(x, \xi) \log ^{k-1}|\xi|+\cdots+a_{0}(x, \xi),
$$

where $k \in \mathbb{N}$ and $a_{0}, \ldots, a_{k}$ are classical symbols. We call the integer $k$ the log-degree of $L$. We denote by:

- $\quad \mathcal{L}$ the algebra of log-polyhomogeneous PDOs acting on smooth sections of $E$;

- $\quad C \ell$ the subalgebra of classical PDOs in $\mathcal{L}$;

- $\quad Q$ an admissible classical PDO of positive order such that $\log Q$ exists;

(C) 2011 Australian Mathematical Publishing Association Inc. 1446-7887/2011 \$16.00 
- $\quad \mathcal{A}_{C \ell}$ a subalgebra of $C \ell$ such that $\left[\mathcal{A}_{C \ell}, \log Q\right] \subset \mathcal{A}_{C \ell}$ (in general, we only know that the commutator of a classical PDO and a logarithm is a classical PDO; hence, we only have $\left.\left[\mathcal{A}_{C \ell}, \log Q\right] \subset C \ell\right)$;

- $\quad \mathcal{A}$ the subalgebra of $\mathcal{L}$ generated by $\log Q$ and $\mathcal{A}_{C \ell}$.

The assumption on $\mathcal{A}_{C \ell}$ implies the following fundamental decomposition of $\mathcal{A}$ (see Lemma 2.2).

$$
\mathcal{A}=\bigoplus_{k=0}^{+\infty} \mathcal{A}_{C \ell} \log ^{k} Q
$$

We assume that $\mathcal{A}_{C \ell}$ does not consist only of smoothing operators. Otherwise, $\mathcal{A}_{C \ell}$ and $\mathcal{A}$ are algebras of smoothing operators. Guillemin [4] has shown that the $L^{2}$ trace is the unique trace on such algebras.

We say that a linear form $\tau$ on an algebra is a trace if, for any operators $A$ and $B$ in the algebra, we have $\tau(A B)=\tau(B A)$. That is, $\tau$ vanishes on commutators. On a graded algebra $\mathcal{B}=\bigoplus_{k \geq 0} \mathcal{B}^{k}$, a graded trace is a sequence $\left(\tau_{k}\right)_{k \in \mathbb{N}}$ of linear forms $\tau_{k}$ on $\bigoplus_{0 \leq l \leq k} \mathcal{B}^{l}$ which vanishes on $\bigoplus_{0 \leq l \leq k-1} \mathcal{B}^{l}$ and satisfies $\tau_{k+m}(A B)=\tau_{k+m}(B A)$ for $A \in \bigoplus_{0 \leq l \leq k} \mathcal{B}^{l}$ and $B \in \bigoplus_{0 \leq l \leq m} \overline{\mathcal{B}}^{l}$.

Under the assumption of the uniqueness of a trace $\tau_{0}$ on $\mathcal{A}_{C \ell}$, we show that there exists a unique graded trace $\left(\bar{\tau}_{0}{ }^{k}\right)_{k \in \mathbb{N}}$ on the whole algebra $\mathcal{A}$ which extends $\tau_{0}$ (see Theorem 3.3). We also prove that, if there exists a trace on $\mathcal{A}$ extending $\tau_{0}$, then this extension is unique (see Theorem 3.5).

Our first result applies to the Wodzicki-Guillemin residue (also called the noncommutative residue) Res on the algebra $C \ell$ provided that $M$ is of dimension $n \geq 2$. It is well known that the Wodzicki-Guillemin residue Res is the unique trace on $C \ell$. Setting $\operatorname{Res}_{0}=\operatorname{Res}$, the extension $\overline{\operatorname{Res}}_{0}^{k}$ coincides, up to a multiplicative factor, with the higher noncommutative residue Res ${ }_{k}$ introduced by Lesch.

The $k$ th residue of an operator $L$ in $\mathcal{L}$ of $\log$-degree $k$ with local symbol

$$
\sigma(L)=a_{k}(x, \xi) \log ^{k}|\xi|+a_{k-1}(x, \xi) \log ^{k-1}|\xi|+\cdots+a_{0}(x, \xi)
$$

is defined by

$$
\operatorname{Res}_{k}(L)=(k+1) ! \int_{M} \int_{S^{*} M} \operatorname{tr}\left(\left(a_{k}\right)_{-n}(x, \xi)\right) d \xi d x .
$$

When $\mathcal{L}$ is seen as a graded algebra (the grading is given by log-degrees), Lesch has shown in [8] that the sequence $\left(\operatorname{Res}_{k}\right)_{k \in \mathbb{N}}$ is the unique graded trace. We recover the same result by an alternative approach.

Our second result applies to the canonical trace on the algebra of odd-class logpolyhomogeneous PDOs when the manifold $M$ is odd dimensional. According to Kontsevitch and Vishik [6,7], a classical operator $A$ of order $m \in \mathbb{Z}$ is of odd class if, locally, the positively homogeneous components of its symbol $\left\{a_{m-j} \mid j \in \mathbb{Z}\right\}$ are simply homogeneous, that is, they have the property that

$$
a_{m-j}(x,-\xi)=(-1)^{m-j} a_{m-j}(x, \xi) .
$$


The odd-class classical PDOs form an algebra. Following [10], we say that a $\log$-polyhomogeneous PDO $L$ is of odd class if, locally, all the classical symbols $a_{0}, \ldots, a_{k}$ arising as coefficients of powers of $\log |\xi|$ in its symbol (see (1.1)) have the above property (1.3). In a similar manner to that for odd-class classical PDOs, one can easily check that odd-class log-polyhomogeneous PDOs form an algebra.

When $M$ is odd dimensional, the canonical trace TR of [6] is well defined on the algebra of odd-class classical PDOs. Recently, Maniccia et al. [9] proved that TR is the unique trace on this algebra. The canonical trace was first extended by Lesch [8] to log-polyhomogeneous PDOs of noninteger orders (this means that the orders of $a_{0}, \ldots, a_{k}$ are not integers). It has further been extended by Paycha and Scott [10] to odd-class log-polyhomogeneous PDOs when $M$ is odd dimensional. We refer the reader to Section 4 for more details. To the best of our knowledge, our proof of the uniqueness of TR on odd-class log-polyhomogeneous PDOs is new.

\section{Preliminaries}

Following [6, 12, 13], we begin by briefly reviewing the definitions of complex powers and then those of logarithms of an operator of positive order.

Let $Q$ be an invertible classical PDO of positive order $q$. We say that $Q$ is admissible if there exists a closed angle of vertex zero which does not intersect the spectrum of the leading symbol of $Q$. If $Q$ is admissible, then there is a half-line

$$
L_{\theta}=\{z \in \mathbb{C} \mid \arg z=\theta\}
$$

which does not meet the spectrum of $Q$. We call such a half-line a spectral cut.

Now let $Q$ be an admissible PDO with spectral cut $L_{\theta}$. Then complex powers $Q_{\theta}^{s}$ are well defined for all $s \in \mathbb{C}$ and the logarithm $\log _{\theta} Q$ is obtained as the derivative at zero $\log _{\theta} Q=\left.D_{s} Q_{\theta}^{s}\right|_{s=0}$. The complex power $Q_{\theta}^{s}$ is still an invertible classical PDO of order $q s$, whereas $\log _{\theta} Q$ is no longer classical. Locally, its symbol reads

$$
q \log |\xi| \mathrm{id}+\sigma_{0}(x, \xi),
$$

where $\sigma_{0}$ is a classical symbol of order zero. Thus, $\log _{\theta} Q$ is a PDO of positive order $\varepsilon$ for all $\varepsilon>0$. Since the choice of a spectral cut will not be important when taking an admissible operator, we will omit the mention of $\theta$.

From now on, let $Q$ be an admissible operator and let $\mathcal{A}_{C \ell}$ be an algebra of classical PDOs which are not reduced to smoothing operators and such that $\left[\mathcal{A}_{C \ell}, \log Q\right]$ lies in $\mathcal{A}_{C \ell}$. Let $\mathcal{A}$ be the algebra generated by $\log Q$ and $\mathcal{A}_{C \ell}$.

The following are some elementary, yet fundamental, results that we shall require in the sequel.

Lemma 2.1. If $A$ is in $\mathcal{A}_{C \ell}$ and if $k \geq 1$, then $\left[A, \log ^{k} Q\right]$ is in $\mathcal{A}$ and is of log-degree $k-1$.

Proof. The fact that $[A, \log Q]$ is classical is stated in [3]. For the sake of completeness, here are the detailed calculations that prove this fact. We recall the 
composition law of symbols corresponding to the composition of PDOs (see [13]):

$$
\sigma \star \sigma^{\prime}(x, \xi)=\sum_{\alpha \in \mathbb{N}^{n}} \frac{(-i)^{|\alpha|}}{\alpha !} \partial_{\xi}^{\alpha} \sigma(x, \xi) \partial_{x}^{\alpha} \sigma^{\prime}(x, \xi) .
$$

Locally, let $\sigma$ be a symbol of $A$ and let

$$
q \log |\xi| \mathrm{id}+\sigma_{0}(x, \xi)
$$

be a symbol of $\log Q$ (as above, $q$ denotes the order of $Q$ ). Then a symbol of $A \log Q$ is

$$
\begin{gathered}
\sigma(A \log Q)(x, \xi)=\sum_{\alpha \in \mathbb{N}^{n}} \frac{(-i)^{|\alpha|}}{\alpha !} \partial_{\xi}^{\alpha} \sigma(x, \xi) \partial_{x}^{\alpha}\left(q \log |\xi| \mathrm{id}+\sigma_{0}(x, \xi)\right), \\
\sigma(A \log Q)(x, \xi)=\left(\sigma \star \sigma_{0}\right)(x, \xi)+q \log |\xi| \sigma(x, \xi)
\end{gathered}
$$

and a symbol of $\log Q A$ is

$$
\begin{aligned}
& \sigma(\log Q A)(x, \xi)= \sum_{\alpha \in \mathbb{N}^{n}} \frac{(-i)^{|\alpha|}}{\alpha !} \partial_{\xi}^{\alpha}\left(q \log |\xi| \operatorname{id}+\sigma_{0}(x, \xi)\right) \partial_{x}^{\alpha} \sigma(x, \xi), \\
& \sigma(\log Q A)(x, \xi)=\left(\sigma_{0} \star \sigma\right)(x, \xi)+q \log |\xi| \sigma(x, \xi) \\
&+q \sum_{\alpha \in \mathbb{N}^{n} \backslash\{0\}} \frac{(-i)^{|\alpha|}}{\alpha !} \partial_{\xi}^{\alpha} \log |\xi| \partial_{x}^{\alpha} \sigma(x, \xi) .
\end{aligned}
$$

The derivative $\partial_{\xi}^{\alpha} \log |\xi|$ is homogeneous of degree $-|\alpha|$. Thus, a symbol of $[A, \log Q]=A \log Q-\log Q A$ is classical. By our assumption on $\mathcal{A}_{C \ell}$, we have $[A, \log Q] \in 0 \mathcal{A}_{C \ell}$.

The general result can be proved by induction on $k$, since we have

$$
\begin{array}{r}
A \log ^{k} Q-\log ^{k} Q A=\left(A \log ^{k-1} Q-\log ^{k-1} Q A\right) \log Q \\
+\log ^{k-1} Q A \log Q-\log ^{k} Q A,
\end{array}
$$

$$
\left[A, \log ^{k} Q\right]=\left[A, \log ^{k-1} Q\right] \log Q+\log ^{k-1} Q[A, \log Q] .
$$

LEMMA 2.2. If $A \in \mathcal{A}$ has log-degree $k$, then there exist classical PDOs $A_{0}, A_{1}, \ldots, A_{k}$ in $\mathcal{A}_{C \ell}$ such that

$$
A=A_{0}+A_{1} \log Q+\cdots+A_{k} \log ^{k} Q .
$$

That is,

$$
\mathcal{A}=\bigoplus_{k=0}^{+\infty} \mathcal{A}_{C \ell} \log ^{k} Q
$$

Proof. By definition of $\mathcal{A}, A$ is a linear combination of terms of the form

$$
C_{1} \log ^{\alpha_{1}} Q C_{2} \log ^{\alpha_{2}} Q \cdots C_{m} \log ^{\alpha_{m}} Q C_{m+1},
$$


where the $C_{i}$ are in $\mathcal{A}_{C \ell}$ and the $\alpha_{i}$ are nonnegative integers which sum up to $k$. We notice that

$$
\log ^{\alpha_{m}} Q C_{m+1}=C_{m+1} \log ^{\alpha_{m}} Q+\left[\log ^{\alpha_{m}} Q, C_{m+1}\right] .
$$

Hence, by induction on $\alpha_{m}$ and Lemma 2.1, there exist classical PDOs $A_{m+1,0}$, $A_{m+1,1}, \ldots, A_{m+1, \alpha_{m-1}}$ in $\mathcal{A}_{C \ell}$ such that

$$
\begin{aligned}
\log ^{\alpha_{m}} Q C_{m+1}= & C_{m+1} \log ^{\alpha_{m}} Q+A_{m+1,0} \\
& +A_{m+1,1} \log Q+\cdots+A_{m+1, \alpha_{m-1}} \log ^{\alpha_{m-1}} Q .
\end{aligned}
$$

The result follows by induction on $m$.

Proposition 2.3. Let

$$
A=A_{0}+A_{1} \log Q+\cdots+A_{k} \log ^{k} Q \in \mathcal{A}
$$

be an operator of log-degree $k$. Then:

(1) the classical PDO $A_{k}$ is unique up to a smoothing operator;

(2) if there exists a trace $\tau_{0}$ on $\mathcal{A}_{C \ell}$ which vanishes on smoothing operators, then the linear form ${\overline{\tau_{0}}}^{k}: A \mapsto \tau_{0}\left(A_{k}\right)$ defines a graded trace on $\mathcal{A}$.

PROOF. For part (1), let us consider an alternative decomposition of $A$, namely

$$
A=A_{0}^{\prime}+A_{1}^{\prime} \log Q+\cdots+A_{k}^{\prime} \log ^{k} Q .
$$

The two decompositions lead to two descriptions of the local symbol of $A$ :

$$
\sigma(A)=q^{k} \sigma\left(A_{k}\right) \log ^{k}|\xi|+\sigma
$$

and

$$
\sigma(A)=q^{k} \sigma\left(A_{k}^{\prime}\right) \log ^{k}|\xi|+\sigma^{\prime}
$$

where $\sigma\left(A_{k}\right)$ and $\sigma\left(A_{k}^{\prime}\right)$ are symbols of $A_{k}$ and $A_{k}^{\prime}$, respectively, $\sigma$ and $\sigma^{\prime}$ are logpolyhomogeneous symbols of $\log$-degree $k-1$ and $q$ is the order of $Q$. It follows that the classical symbols $\sigma\left(A_{k}\right)$ and $\sigma\left(A_{k}^{\prime}\right)$ differ from smoothing symbols. This implies that the difference $A_{k}-A_{k}^{\prime}$ is a smoothing operator.

In part (2), if $\tau_{0}$ vanishes on smoothing operators, then, since $A_{k}$ is unique modulo a smoothing operator, the linear form ${\overline{\tau_{0}}}^{k}$ is well defined. Let $A$ and $B$ be two $\log$ polyhomogeneous PDOs written as

$$
A=A_{0}+A_{1} \log Q+\cdots+A_{k} \log ^{k} Q
$$

and

$$
B=B_{0}+B_{1} \log Q+\cdots+B_{m} \log ^{m} Q
$$

We have

$$
{\overline{\tau_{0}}}^{k+m}(A B)={\overline{\tau_{0}}}^{k+m}\left(A_{k} \log ^{k} Q B_{m} \log ^{m} Q\right) .
$$


Using (2.1), we permute $\log ^{k} Q$ and $B_{m}$ to obtain

$$
{\overline{\tau_{0}}}^{k+m}(A B)={\overline{\tau_{0}}}^{k+m}\left(A_{k} B_{m} \log ^{k+m} Q\right) .
$$

This implies that $\bar{\tau}_{0}{ }^{k+m}(A B)=\tau_{0}\left(A_{k} B_{m}\right)$. A similar argument allows us to prove that $\bar{\tau}_{0}^{k+m}(B A)=\tau_{0}\left(B_{m} A_{k}\right)$. The result follows.

\section{Results and proofs}

For $k \geq 0$, we let $\mathcal{A}^{k}$ denote the vector space of operators in $\mathcal{A}$ of log-degree $k$.

LEMMA 3.1. If there exists a unique nontrivial trace $\tau_{0}$ on $\mathcal{A}_{C \ell}$ and if $A \in \mathcal{A}^{k}$, then, for any operator $P$ in $\mathcal{A}_{C \ell}$ such that $\tau_{0}(P)=1$, there exists a finite number of operators $P_{i}$ in $\mathcal{A}_{C \ell}, Q_{i}$ in $\mathcal{A}^{k}$ and a finite number of complex scalars $\alpha_{i}$ such that

$$
A=\sum_{i=1}^{M}\left[P_{i}, Q_{i}\right]+P\left(\alpha_{0}+\alpha_{1} \log Q+\cdots+\alpha_{k} \log ^{k} Q\right) .
$$

In particular, $\alpha_{k}=\tau_{0}\left(A_{k}\right)$ when $A$ is written as

$$
A=A_{0}+A_{1} \log Q+\cdots+A_{k} \log ^{k} Q .
$$

If $\mathcal{A}_{C \ell}$ does not admit any nontrivial trace, then every $A \in \mathcal{A}^{k}$ can be written in the form

$$
A=\sum_{i=1}^{M}\left[P_{i}, Q_{i}\right]
$$

with $P_{i}$ in $\mathcal{A}_{C \ell}$ and $Q_{i}$ in $\mathcal{A}^{k}$.

PROOF. We proceed by induction on $k$.

Let $k=0$. In this case, $A$ belongs to $\mathcal{A}_{C \ell}$. Let us assume that there exists a unique nontrivial trace $\tau_{0}$ on $\mathcal{A}_{C \ell}$. Let $P \in \mathcal{A}_{C \ell}$ be such that $\tau_{0}(P)=1$.

Let $\mathcal{D}$ be the vector subspace of $\mathcal{A}_{C \ell}$ generated by the commutators of $\mathcal{A}_{C \ell}$ and let $\mathcal{D}^{\perp}$ be the orthogonal of $\mathcal{D}$ in the algebraic dual space of $\mathcal{A}_{C \ell}$. By definition, $\mathcal{D}^{\perp}$ is the set of linear forms which vanish on $\mathcal{D}$. Hence, a trace on $\mathcal{A}_{C \ell}$ is an element of the subspace $\mathcal{T}=\mathcal{D}^{\perp}$ and the assumption of the uniqueness of $\tau_{0}$ implies that $\mathcal{T}$ is generated by $\tau_{0}$. Now it is a general fact that any vector subspace $F$, of finite or infinite dimension, of a vector space $\mathcal{E}$ satisfies $\left(F^{\perp}\right)^{\perp}=F$. Here $\left(F^{\perp}\right)^{\perp}$ is the vector subspace of $\mathcal{E}$ orthogonal to $F^{\perp}$ for the pairing between $\mathcal{E}$ and its dual (see, for example, $\left[1\right.$, Section 7 , no. 5, Theorem 7]). Hence, we have $\left(\mathcal{D}^{\perp}\right)^{\perp}=\mathcal{D}$. That is, $\mathcal{T}^{\perp}=\mathcal{D}$. But, by definition of

$$
\mathcal{T}^{\perp}=\left\{A \in \mathcal{A}_{C \ell} \mid \tau_{0}(A)=0\right\}
$$

we have $\mathcal{T}^{\perp}=\operatorname{Ker} \tau_{0}$. Hence, $\mathcal{D}=\operatorname{Ker} \tau_{0}$ is of codimension one. This leads to the following decomposition of $A$ :

$$
A=\sum_{i=1}^{M}\left[P_{i}, Q_{i}\right]+\tau_{0}(A) P
$$


with $P_{i}$ and $Q_{i}$ in $\mathcal{A}_{C \ell}$ for all $i$. Otherwise, if $\tau_{0}=0$, we simply have that $A=\sum_{i=1}^{M}\left[P_{i}, Q_{i}\right]$.

Suppose that property (3.1) holds for some $k \geq 0$. Let $A \in \mathcal{A}^{k+1}$ and let the elements $A_{0}, \ldots, A_{k+1}$ of $\mathcal{A}_{C \ell}$ be such that

$$
A=A_{0}+A_{1} \log Q+\cdots+A_{k+1} \log ^{k+1} Q .
$$

Since $A_{k+1}$ lies in $\mathcal{A}_{C \ell}$, the case for $k=0$ gives us the decomposition

$$
A_{k+1}=\sum_{i=1}^{N}\left[P_{k+1, i}, Q_{k+1, i}\right]+\tau_{0}\left(A_{k+1}\right) P
$$

with $P_{k+1, i}$ and $Q_{k+1, i}$ in $\mathcal{A}_{C \ell}$ for all $i$.

In any algebra, we have that

$$
[X, Y] Z=[X, Y Z]+Y[Z, X] .
$$

Thus,

$$
\begin{aligned}
A_{k+1} \log ^{k+1} Q= & \left(\sum_{i=1}^{N}\left[P_{k+1, i}, Q_{k+1, i}\right]+\tau_{0}\left(A_{k+1}\right) P\right) \log ^{k+1} Q \\
= & \sum_{i=1}^{N}\left[P_{k+1, i}, Q_{k+1, i} \log ^{k+1} Q\right]+\sum_{i=1}^{N} Q_{k+1, i}\left[\log ^{k+1} Q, P_{k+1, i}\right] \\
& \quad+\tau_{0}\left(A_{k+1}\right) P \log ^{k+1} Q .
\end{aligned}
$$

Now $\left[P_{k+1, i}, Q_{k+1, i} \log ^{k+1} Q\right]$ is a commutator with $P_{k+1, i}$ in $\mathcal{A}_{C \ell}$ and $Q_{k+1, i} \log ^{k+1} Q$ in $\mathcal{A}^{k+1}$. By Lemma 2.1, $Q_{k+1, i}\left[\log ^{k+1} Q, P_{k+1, i}\right]$ is of log-degree $k$ so that we can apply the inductive hypothesis to $\sum_{i=1}^{N} Q_{k+1, i}\left[\log ^{k+1} Q, P_{k+1, i}\right]$. We may also apply the inductive hypothesis to

$$
A_{0}+A_{1} \log Q+\cdots+A_{k} \log ^{k} Q .
$$

Property (3.1) follows.

Suppose that property (3.2) holds and $\tau_{0}=0$. We may deduce from our calculations that, if $A$ is in $\mathcal{A}^{k+1}$, then $A$ is a sum of commutators of operators in $\mathcal{A}_{C \ell}$ and $\mathcal{A}^{k+1}$.

REMARK 3.2. Lemma 3.1 extends to several traces on $\mathcal{A}_{C \ell}$. If there exist $m$ linearly independent traces $\tau_{0}, \ldots, \tau_{m}$ on $\mathcal{A}_{C \ell}$, then there exist $m$ operators $\widetilde{P}_{0}, \ldots, \widetilde{P_{m}}$ in $\mathcal{A}_{C \ell}$ and scalars $\alpha_{l, j}, 0 \leq l \leq k, 0 \leq j \leq m$ such that $\tau_{j}\left(\widetilde{P}_{i}\right)=\delta_{i j}$ and

$$
A=\sum_{i=1}^{M}\left[P_{i}, Q_{i}\right]+\sum_{j=1}^{m} \widetilde{P}_{j}\left(\alpha_{0, j}+\alpha_{1, j} \log Q+\cdots+\alpha_{k, j} \log ^{k} Q\right) .
$$

PROOF. In this case, the vector subspace $\mathcal{D}$ of $\mathcal{A}_{C \ell}$ generated by commutators satisfies $\mathcal{D}=\bigcap_{i=1}^{m} \operatorname{Ker} \tau_{i}$. Hence, $\mathcal{D}$ is of codimension $m$ in $\mathcal{A}_{C \ell}$. The proof is similar to that for Lemma 3.1. 
THEOREM 3.3. Suppose that there exists a unique nontrivial trace $\tau_{0}$ on $\mathcal{A}_{C \ell}$. If $\tau_{0}$ vanishes on smoothing operators, then the graded trace $\left(\bar{\tau}_{0}^{k}\right)_{k \in \mathbb{N}}$ on $\mathcal{A}$ extending $\tau_{0}$ is unique up to a multiplicative factor (depending on $k$ ). In contrast, there is no nontrivial trace on $\mathcal{A}$.

Proof. Let $A \in \mathcal{A}$ be of $\log$-degree $k \geq 1$. By Lemma 2.2, $A$ can be written as

$$
A=A_{0}+A_{1} \log Q+\cdots+A_{k} \log ^{k} Q
$$

with the $A_{i}$ in $\mathcal{A}_{C \ell}$. Let us assume that $\tau_{0}$ is nontrivial.

Applying Lemma 3.1, for any $P$ in $\mathcal{A}_{C \ell}$ such that $\tau_{0}(P)=1$ we have

$$
A=\sum_{i=1}^{M}\left[P_{i}, Q_{i}\right]+L_{k}
$$

with

$$
L_{k}=P\left(\alpha_{0}+\alpha_{1} \log Q+\cdots+\alpha_{k-1} \log ^{k-1} Q+\tau_{0}\left(A_{k}\right) \log ^{k} Q\right),
$$

where the $P_{i}$ are in $\mathcal{A}_{C \ell}$, the $Q_{i}$ are in $\mathcal{A}^{k}$ and the $\alpha_{i}$ are complex numbers.

Suppose that $\tau_{0}$ vanishes on smoothing operators. We can choose $P$ to be nonsmoothing. Let $\left(\tau_{k}\right)_{k \in \mathbb{N}}$ be a graded trace on $\mathcal{A}$. Then we have

$$
\tau_{k}(A)=\tau_{k}\left(L_{k}\right)=\tau_{k}\left(P \log ^{k} Q\right) \tau_{0}\left(A_{k}\right) .
$$

Since $P$ is independent of $A, \tau_{k}(A)$ is equal to $\tau_{0}\left(A_{k}\right)$ up to a multiplicative factor which is independent of $k$. Using the notation of Proposition 2.3, we have $\tau_{0}\left(A_{k}\right)=$ $\bar{\tau}_{0}^{k}(A)$. The uniqueness of $\left({\overline{\tau_{0}}}^{k}\right)_{k \in \mathbb{N}}$ follows.

Now suppose that there exists a trace $\tau$ extending $\tau_{0}$ to $\mathcal{A}$. To conclude that $\tau$ is trivial, we use an argument of Lesch (see [8]). For $A \in \mathcal{A}$ of log-degree $k$, we have $\bar{\tau}_{0}^{k+1}(A)=0$. By the uniqueness of $\left(\bar{\tau}_{0}^{k}\right)_{k \in \mathbb{N}}, A$ is a sum of commutators $\left[P_{i}, Q_{i}\right]$ with $P_{i}$ in $\mathcal{A}_{C \ell}$ and $Q_{i}$ in $\mathcal{A}^{k+1}$. Hence, $A$ is a sum of commutators and $\tau(A)=0$.

REMARK 3.4. If there exist $m$ linearly independent traces $\tau_{0}, \ldots, \tau_{m}$ on $\mathcal{A}_{C \ell}$ which all vanish on smoothing operators, then any graded trace on $\mathcal{A}$ is a linear combination of the $m$ extensions $\left(\bar{\tau}_{0}^{k}\right)_{k \in \mathbb{N}}, \ldots,\left(\bar{\tau}_{m}^{k}\right)_{k \in \mathbb{N}}$.

PROOF. The result is a straightforward application of Remark 3.2 to the proof of Theorem 3.3.

THEOREM 3.5. Suppose that there exists a unique nontrivial trace $\tau_{0}$ on $\mathcal{A}_{C \ell}$. If $\tau_{0}$ does not vanish on smoothing operators and if there exists a trace $\tau$ extending $\tau_{0}$ to $\mathcal{A}$, then $\tau$ is unique.

PROOF. Suppose that $\tau_{0}$ is nontrivial, but does not vanish on smoothing operators. We begin as in the proof of Theorem 3.3. In formula (3.3), we can choose $P$ to be smoothing and satisfying $\tau_{0}(P)=1$. Since $P$ is smoothing, $L_{k}$ is also smoothing. Now let $\operatorname{Tr}$ be the unique trace on smoothing operators. It follows that there exists $\beta$ in $\mathbb{C}^{*}$, independent of $A$ and such that $\tau(A)=\tau\left(L_{k}\right)=\beta \operatorname{Tr}\left(L_{k}\right)$. This implies the uniqueness of the trace $\tau$ on $\mathcal{A}$. 
REMARK 3.6. If $\tau_{0}=0$, then any graded trace or ordinary trace extending $\tau_{0}$ on $\mathcal{A}$ vanishes. Notice also that, if $\tau_{0}$ does not vanish on smoothing operators, then any graded trace on $\mathcal{A}$ extending $\tau_{0}$ vanishes on $\mathcal{A} \backslash \mathcal{A}_{C \ell}$.

PROOF. If $\tau_{0}=0$, then by Lemma $3.1 A$ is a sum of commutators of operators. The first result follows.

Now let us assume that $\tau_{0}$ does not vanish on smoothing operators, and let $\left(\tau_{k}\right)_{k \in \mathbb{N}}$ be a graded trace extending $\tau_{0}$. Following the proof of Theorem 3.5, $\tau_{k}$ should be proportional to the trace of a smoothing operator for any $k \geq 0$. Hence, $\tau_{k}=0$ for all $k \geq 1$.

\section{Applications}

We let $\mathcal{L}_{\text {odd }}$ denote the algebra of odd-class log-polyhomogeneous PDOs and let $C \ell_{\text {odd }}$ denote the algebra of odd-class classical PDOs. The following proposition says that both $\mathcal{L}$ and $\mathcal{L}_{\text {odd }}$ satisfy property (1.2) of the introduction. We begin with a lemma about the odd-parity class of a logarithm, which can be found in [6] or [2].

LEMMA 4.1. Let $Q$ be an admissible operator. If $Q$ is of odd class and even order, then $\log Q$ is of odd class.

PROOF. Let $Q$ be of odd class and of even order $q$. We recall that $Q^{s}$ is a classical PDO of order $q s$. We will denote by $\sigma_{q-j}$, for $j \geq 0$, the homogeneous components of a symbol of $Q$. For $s \in \mathbb{C}$ of negative real part and for a suitable contour $\Gamma$ (see [12]), the positively homogeneous components of $Q^{s}$ of degree $q s-j$ are expressed by

$$
\sigma_{q s-j}(x, \xi)=\frac{i}{2 \pi} \int_{\Gamma} \lambda^{s} b_{-q-j}(x, \xi, \lambda) d \lambda
$$

with $b_{-q}=\left(\sigma_{q}-\lambda \mathrm{Id}\right)^{-1}$ and satisfying, for $j \geq 1$,

$$
b_{-q-j}=-b_{-q} \sum_{k+l+|\alpha|=j, l<j} i^{-|\alpha|} \frac{1}{\alpha !} \partial_{\xi}^{\alpha} \sigma_{q-k} \partial_{x}^{\alpha} b_{-q-l} .
$$

We have

$$
b_{-q}(x,-\xi, \lambda)=\left(\sigma_{q}(x,-\xi)-\lambda \operatorname{Id}(x, \xi)\right)^{-1} .
$$

Since $Q$ is of odd class and $q$ is even, we have $b_{-q}(x,-\xi, \lambda)=b_{-q}(x, \xi, \lambda)$. By induction on $j$, we deduce that

$$
b_{-q-j}(x,-\xi, \lambda)=(-1)^{j} b_{-q-j}(x, \xi, \lambda) .
$$

Thus, we have

$$
\sigma_{q s-j}(x,-\xi)=(-1)^{j} \sigma_{q s-j}(x, \xi) .
$$

For $s=0$, we have $A^{0}=\operatorname{Id}$ which is odd and for $\operatorname{Re}(s)>0$ we use $A^{s} A^{-s}=\operatorname{Id}$ to conclude that this equality still holds. Observing that

$$
\sigma_{q s-j}(x, \xi)=|\xi|^{q s-j} \sigma_{q s-j}(x, \xi /|\xi|)
$$

and differentiating at $s=0$ gives us that $\log Q$ is of odd class. 
PROPOSITION 4.2. Let $Q$ be an admissible operator. Then:

(1) $\mathcal{L}=\bigoplus_{k=0}^{+\infty} C \ell \log ^{k} Q ;$

(2) if $Q$ is an admissible operator of odd class and of even order, then $\log Q$ is of odd class and

$$
\mathcal{L}_{\text {odd }}=\bigoplus_{k=0}^{+\infty} C \ell_{\text {odd }} \log ^{k} Q .
$$

PROOF. In part (1), the inclusion from right to left is straightforward. To show the inclusion from left to right, we proceed by induction on $k$. The inclusion certainly holds for $k=0$. Suppose that it holds for some $k \geq 0$. Let $A \in \mathcal{A}$ be of log-degree $k+1$ with symbol $a=\sum_{l=0}^{k+1} a_{l} \log ^{l}|\xi|$. Using a partition of unity adapted to a finite trivializing covering of $M$ for $E$, we associate to $a_{k+1}$ a classical operator

$$
A_{k+1}=O p\left(a_{k+1}\right) \text {. }
$$

Then the operator

$$
A-\frac{1}{q^{k+1}} A_{k+1}\left(\log ^{k+1} Q\right)
$$

lies in $\mathcal{A}$ and is of log-degree $k$.

The result follows by our inductive hypothesis.

The proof of part (2) proceeds like that of part (1) with $A_{k+1}$ and $\log ^{k+1} Q$ of odd class.

Now we recall the definition of the canonical trace on the algebra $\mathcal{L}_{\text {odd }}$ when $M$ is odd dimensional. For this definition, we follow [10] (see also [8]).

Let $L$ be in $\mathcal{L}_{\text {odd }}$ of log-degree $k$ with local symbol $\sigma=\sum_{l=0}^{k} a_{l} \log ^{l}|\xi|$. The canonical trace of $L$ is defined by

$$
\operatorname{TR}(L)=\int_{M} \operatorname{TR}_{x}(L) d x
$$

via a well-defined global density on $M$

$$
\operatorname{TR}_{x}(L) d x=\left(f_{T_{x}^{*} M} \operatorname{tr}_{x}(\sigma(x, \xi)) d \xi\right) d x .
$$

Here the finite part integral $f_{T_{x}^{*} M} \operatorname{tr}_{x}(\sigma(x, \xi)) d \xi$ is the constant term in the asymptotic expansion of $\int_{|\xi|<R} \operatorname{tr}_{x}(\sigma(x, \xi)) d \xi$ when $R \rightarrow+\infty$.

On smoothing operators, the canonical trace coincides with the $L^{2}$ trace, whereas the Wodzicki-Guillemin residue clearly vanishes.

APPLICATION 4.3. When the manifold is of dimension $\geq 2$, we know from Wodzicki (see [5, 14]) and Guillemin (see [4]) that Res is the unique trace on $C \ell$ (see also [11] for detailed analysis on this subject). When combined with Proposition 4.2, Theorem 3.3 gives the existence and the uniqueness of $\left(\operatorname{Res}_{k}\right)_{k \in \mathbb{N}}$ on $\mathcal{L}$. 
Application 4.4. When we combine Proposition 4.2 with the result of [9], the uniqueness of TR on odd-class classical PDOs for odd-dimensional manifolds and Theorem 3.5, we obtain a proof of the uniqueness of TR on $\mathcal{L}_{\text {odd }}$ in odd dimensions.

\section{References}

[1] N. Bourbaki, 'Algèbre linéaire', in: Algèbre, 3rd edn (Hermann, Paris, 1962), Ch. 2.

[2] M. Braverman, 'Symmetrized trace and symmetrized determinant of odd class pseudo-differential operators', J. Geom. Phys. 59(4) (2009), 459-474.

[3] A. Cardona, C. Ducourtioux, J.-P. Magnot and S. Paycha, 'Weighted traces on algebras of pseudodifferential operators and geometry on loop groups', Infin. Dimens. Anal. Quantum Probab. Relat. Top. 5 (2002), 503-540.

[4] V. Guillemin, 'Residue traces for certain algebras of Fourier integral operators', J. Funct. Anal. 115 (1993), 391-417.

[5] C. Kassel, 'Le résidu non commutatif (d'après M. Wodzicki)' (in French) [The noncommutative residue (after M. Wodjicki)] Séminaire Bourbaki, Vol. 1988/89. Astérisque No. 177-178 (1989), Exp. No. 708, 199-229.

[6] M. Kontsevich and S. Vishik, 'Geometry of determinants of elliptic operators', in: Functional Analysis on the Eve of the XXI Century, Vol. I, Progress in Mathematics, 131 (Birkhäuser, Boston, MA, 1995), pp. 173-197.

[7] M. Kontsevich and S. Vishik, 'Determinants of elliptic pseudodifferential operators', Max Planck Preprint, 1994.

[8] M. Lesch, 'On the noncommutative residue for pseudodifferential operators with logpolyhomogeneous symbols', Ann. Global Anal. Geom. 17 (1998), 151-187.

[9] L. Maniccia, E. Schrohe and J. Seiler, 'Uniqueness of the Kontsevich-Vishik trace', Proc. Amer. Math. Soc. 136 (2008), 747-752.

[10] S. Paycha and S. Scott, 'A Laurent expansion for regularised integrals of holomorphic symbols', Geom. Funct. Anal. 17 (2007), 491-536.

[11] R. Ponge, 'Traces on pseudodifferential operators and sums of commutators', J. Anal. Math. 110 (2010), 1-30.

[12] R. T. Seeley, 'Complex powers of an elliptic operator, singular integrals', in: Proceedings of the Symposium in Pure Mathematics, Chicago (American Mathematical Society, Providence, RI, 1966), pp. 288-307.

[13] M. A. Shubin, Pseudodifferential Operators and Spectral Theory, Springer Series in Soviet Mathematics (Springer, Berlin, 1987).

[14] M. Wodzicki, 'Noncommutative residue. I. Fundamentals', in: K-theory, Arithmetic and Geometry (Moscow, 1984-1986), Lecture Notes in Mathematics, 1289 (Springer, New York, 1987), pp. 320-399.

C. DUCOURTIOUX, Département de Mathématiques, Université Pascal Paoli, 20250 Corte, France

e-mail: ducourtioux@univ-corse.fr

M. F. OUEDRAOGO, Département de Mathématiques, Université de Ouagadougou,

03 BP 7021, Burkina Faso

e-mail: marie.oued@univ-ouaga.bf 